\title{
Design of biomolecular network modifications to achieve adaptation
}

\author{
Steffen Waldherr ${ }^{1}$, Stefan Streif ${ }^{2}$, and Frank Allgöwer ${ }^{1}$ \\ ${ }^{1}$ Institute for Systems Theory and Automatic Control, \\ Universität Stuttgart \\ Pfaffenwaldring 9, 70550 Stuttgart, Germany \\ (e-mail: waldherr@ist.uni-stuttgart.de, allgower@ist.uni-stuttgart.de) \\ ${ }^{2}$ Institute for Automation Engineering, \\ Systems Theory and Automatic Control Laboratory, \\ Otto-von-Guericke Universität Magdeburg \\ Universitätsplatz 2, 39106 Magdeburg, Germany \\ (e-mail: stefan.streif@ovgu.de)
}

This paper is a postprint of a paper submitted to and accepted for publication in IET Systems Biology and is subject to Institution of Engineering and Technology Copyright. The copy of record is available at IET Digital Library.

Reference for citation: IET Systems Biology 6(6):223-231, 2012 


\begin{abstract}
A biomolecular network is called adaptive if its output returns to the original value after a transient response even under a persisting stimulus. The conditions for adaptation have been investigated thoroughly with systems theory approaches in the literature and it is easy to check whether they are satisfied in the linear approximation. In contrast, it is in general not easy to modify a non-adaptive network model such that it gains adaptive behaviour, especially for medium and large-scale networks. We present a systematic approach based on the notion of kinetic perturbations to construct adaptive biomolecular network models from non-adaptive ones. An advantage of kinetic perturbations in this application is that neither the stoichiometry nor the steady state of the system is changed. Furthermore, the method covers both parameter and network structure modifications and can be applied to any reaction rate formalism and even to medium-scale or partially unknown models. The approach is exemplified at a small and a medium-sized biomolecular network, illustrating its potential to systematically evaluate the different network modifications for adaptation.

The proposed method will be useful either in iterative model building to construct mathematical models of adaptive biomolecular networks, or in synthetic biology where it can be applied to design or modify synthetic networks for adaptation.
\end{abstract}




\section{Introduction}

Adaptation of a biomolecular network denotes the property that an observed output initially responds to a stimulus, but resets itself to the prestimulus level in the limit of large times, even though the stimulus persists. Adaptation can be found in single cell organisms and also in sensory cells of higher organisms, and it has been observed e. g. in eukaryotic gradient sensing [1], bacterial chemotaxis [2], archaeal phototaxis [3], yeast osmo regulation [4], or MAP kinase activities [5]. In general, adaptation is important to keep cells fit in changing environments by maintaining homeostasis under perturbations, or by expanding the dynamic range of sensory receptors. If the output converges exactly to the prestimulus level, adaptation is often called perfect, and it is called partial if the output only returns close to, but not exactly to the prestimulus level. Adaptation in biomolecular networks often exhibits robustness against network perturbations [6] and may persist for input stimuli varying over several orders of magnitude [7].

From a systems theoretic point of view, the conditions for mathematical models of biomolecular networks to show perfect adaptation have been extensively investigated $[5,8,9,10,11,12]$. It is known from the internal model principle that if a system adapts to a class of input signals, then it necessarily contains a subsystem that is capable of generating signals of this class [9]. For step inputs or slowly varying inputs, this corresponds to an internal integrator. In the linear approximation, this condition is equivalent to the transfer function from the input to the output of the linearized system having a transmission zero at the origin. This condition is easy to check for any given network.

Considering the interaction structure of the network, adaptation can be achieved by certain feedback or feedforward structures [13, 14]. Ma and coworkers [8] argued that adaptation to persisting (step) inputs can be achieved by a negative feedback loop with a buffering node, or incoherent feedforward loop with a proportioner node $[8,9]$.

Even though the interaction structure of a network is an important prerequisite for achieving adaptation, perfect adaptation is unlikely to arise from random choices of parameters for a network possessing the described structures [15, 8]. For certain types of kinetics (usually zero-order kinetics), adaptation can be robust with respect to parameter variations. However, parameter tuning might be necessary for other types such as Michaelis-Menten, where zero-order kinetics are approximatively obtained if the corresponding enzyme works in saturation.

In modeling of systems biology networks, one often faces large networks with dozens of species and reactions. If a model is non-adaptive, adaptation can then potentially be achieved by varying many parameters, interactions, or reaction kinetics. Such a setup will typically not be straightforward to analyze, and it might not be possible or desired to focus on or identify one single network motif responsible for the adaptive behaviour.

In this paper, we propose a method that helps to identify the network interactions that can be modified for achieving adaptation to step inputs. Kinetic perturbations [16] are applied to induce a transmission zero at the origin of the transfer function from the stimulation (input) to the output of the linear approximation. To demonstrate the application of our approach, we present two examples, a simple three enzyme network and a MAPK cascade model. 


\section{Kinetic perturbations for adaptation}

\subsection{Conditions for local adaptation}

We study adaptation for biochemical network models given by equations of the form

$$
\begin{aligned}
\dot{z} & =N_{r} v(x, u) \\
x & =L z+x_{T} \\
y & =K x .
\end{aligned}
$$

Thereby, $x \in \mathbb{R}^{n}$ is the concentration vector for the network's species, and $z \in \mathbb{R}^{r}$ is the vector of independent concentrations. The concentration vector $x$ is computed from $z$ through the conservation relations, using the link matrix $L \in \mathbb{R}^{n \times r}$ and the vector of conserved moieties $x_{T} \in \mathbb{R}^{n}[17]$. The matrix $N_{r} \in \mathbb{R}^{r \times m}$ is the reduced stoichiometric matrix, where each element $(i, j)$ gives the stoichiometric change of the concentration $z_{i}$ under reaction $j$. Additionally, we consider an input $u \in \mathbb{R}$, representing for example a biochemical stimulus, and an output $y \in \mathbb{R}$, representing a variable for which adaptative behaviour is of interest. The output is assumed to depend linearly on the concentrations via the output matrix $K \in \mathbb{R}^{1 \times n}$. The vector $v(x, u) \in \mathbb{R}^{m}$ is the reaction rate vector. Without conservation relations, the general formulation reduces to the classical network description

$$
\begin{aligned}
\dot{x} & =N v(x, u) \\
y & =K x
\end{aligned}
$$

by setting $L=I_{n}, x_{T}=0$, and $N_{r}=N$.

First, a steady state of the network (1) has to be determined by fixing a nominal input value $u=u_{0}$, setting $\dot{z}$ in (1) to zero, and solving for $z$. The resulting steady state is denoted as the pair $\left(z_{0}, u_{0}\right)$, and also gives rise to the steady state concentration vector $x_{0}=L z_{0}+x_{T}$, output $y_{0}=K x_{0}$, and reaction fluxes $v_{0}=v\left(x_{0}, u_{0}\right)$. The steady state is called exponentially stable, if all eigenvalues of the network's Jacobian evaluated at this steady state have a negative real part.

Definition 1 Let $\left(z_{0}, u_{0}\right)$ be an exponentially stable steady state of (1). Denote by $x(t)$ the concentration time course of (1) with step input $u(t)=u_{0}+u_{s} h(t)$, where $h(t)$ is the unit step, and initial condition $z(0)=z_{0}$. The system (1) is said to perfectly adapt at $\left(z_{0}, u_{0}\right)$, if, for any $u_{s} \in \mathbb{R}$,

$$
\lim _{t \rightarrow \infty}\left(K x(t)-K x_{0}\right)=0 .
$$

It is said to perfectly adapt locally at $\left(z_{0}, u_{0}\right)$, if

$$
\lim _{u_{s} \rightarrow 0} \frac{\lim _{t \rightarrow \infty}\left(K x(t)-K x_{0}\right)}{u_{s}}=0 .
$$

By this definition, "local" adaptation means adaptation to small stimulus changes. Clearly, an infinitesimally small change in the stimulus will yield only an infinitesimally small change in the output for most systems, even for systems that we would consider non-adaptive. The asymptotic gain condition (4), where the output change is divided by the stimulus change, ensures that only systems 
where the output change in the linear approximation goes to zero faster than the stimulus change are classified as adaptive.

Next, we discuss conditions for local adaptation which rely on the network's transfer function in the frequency domain. This transfer function is obtained by a linear approximation to the network's dynamics around the steady state $\left(z_{0}, u_{0}\right)$ and a subsequent Laplace transformation, as discussed in more detail in [18]. Local adaptation of the linear approximation is equivalent to local adaptation of the original network due to local topological equivalence of a non-linear system to its linear approximation at a hyperbolic steady state [19]. The linear approximation is given by the system of linear ordinary differential equations

$$
\begin{aligned}
& \dot{d} z=A d z+B d u \\
& d y=C d z,
\end{aligned}
$$

where $d z, d u$, and $d y$ are infinitesimally small deviations from the steady state variables $z_{0}, u_{0}$, and $y_{0}$, respectively. The matrices $A, B$, and $C$ are computed from

$$
\begin{aligned}
& A=\left.N_{r} \frac{\partial v}{\partial x}\right|_{x_{0}, u_{0}} L \\
& B=\left.N_{r} \frac{\partial v}{\partial u}\right|_{x_{0}, u_{0}} \\
& C=K L .
\end{aligned}
$$

The relation between the output deviation from steady state $d y$ and the stimulus deviation from steady state $d u$ in frequency domain is characterized by the transfer function $G(s)$ computed from the matrices in (6) as

$$
G(s)=C\left(s I_{r}-A\right)^{-1} B,
$$

with the frequency variable $s \in \mathbb{C}$. The transfer function $G$ characterizes the network's gain by the relation $d y(s)=G(s) d u(s)$.

By the final value theorem of the Laplace transformation [20, p. 28], we get the following result: The network (1) has perfect local adaptation at $\left(z_{0}, u_{0}\right)$, if and only if

$$
G(0)=0 .
$$

If $A$ does not have eigenvalues at zero, from the Schur complement formula [20, p. 896], (8) is equivalent to the condition

$$
\operatorname{det}\left(\begin{array}{cc}
A & B \\
C & 0
\end{array}\right)=0 .
$$

For any given biochemical network with conservation relations, the choice of the matrices $L$ and $N_{r}$ in the model (1) is not unique. However, since the property of local adaptation is invariant under state coordinate transformations, conditions (8) and (9) are independent of this choice.

\subsection{Definition and implementation of a kinetic perturba- tion}

A kinetic perturbation is a perturbation of the network's reaction rate vector $v(x, u)$ to become a perturbed reaction rate vector $\tilde{v}(x, u)$. The key restriction 
is that the steady state reaction rates $v_{0}$ should remain unchanged [16]. Other network characteristics, e.g. the stoichiometric matrix, are left unperturbed. Kinetic perturbations are relevant for local adaptation, since they affect the slopes of the reaction rates in steady state. The slopes are mathematically described by the Jacobian $\frac{\partial v}{\partial x}$, and slope changes imply changes to the matrix $A$ in (6), which may affect the condition (9) for local adaptation.

Mathematically, the kinetic perturbations are represented by a dimensionless matrix $\Delta \in \mathbb{R}^{m \times n}$, where the $(i, j)$ entry corresponds to the change in the slope of reaction $i$ with respect to species $j$. The formal definition of a kinetic perturbation is as follows.

Definition 2 A change in the reaction rate expressions in the network (1) from $v$ to $\tilde{v}$ is called a kinetic perturbation, if the following conditions hold:

$$
\begin{aligned}
& \tilde{v}\left(x_{0}, u_{0}\right)=v\left(x_{0}, u_{0}\right) \\
& \left.\frac{\partial \tilde{v}}{\partial u}\right|_{x_{0}, u_{0}}=\left.\frac{\partial v}{\partial u}\right|_{x_{0}, u_{0}} .
\end{aligned}
$$

We also define the perturbation matrix $\Delta \in \mathbb{R}^{m \times n}$ for the kinetic perturbation from $v$ to $\tilde{v}$ as

$$
\Delta=\left(\operatorname{diag} v_{0}\right)^{-1}\left(\left.\frac{\partial \tilde{v}}{\partial x}\right|_{x_{0}, u_{0}}-\left.\frac{\partial v}{\partial x}\right|_{x_{0}, u_{0}}\right) \operatorname{diag} x_{0} .
$$

The normalization of $\Delta$ with $v_{0}$ and $x_{0}$ is also routinely used in metabolic control analysis. A detailed discussion of normalized vs. non-normalized partial derivatives is given in [17, Section 5.7]. There, also the issue of singularities, which occur if $v_{0}$ or $x_{0}$ contain zero elements, is discussed.

The computation of a specific $\Delta$ such that the perturbed network will have local adaptation is described in Section 2.3. Here, we shortly discuss how to construct the perturbed reaction rate vector $\tilde{v}$, given a specific kinetic perturbation $\Delta$ which is to be realized. A direct approach for this task is to perturb $v$ to $\tilde{v}$ by changing some reaction rate parameters [16]. This approach is feasible for certain types of reaction rate laws, such as for example generalized mass action or enzymatic reaction rates. A generalized mass action reaction rate $v_{i}$ is described by the formula

$$
v_{i}(x)=k_{i} \prod_{j=1}^{n} x_{j}^{\sigma_{i j}}
$$

where $k_{i}>0$ is the reaction rate constant and $\sigma_{i j} \in \mathbb{R}$ the kinetic orders of the reaction $i$. A kinetic perturbation can be realised for such a rate by perturbing it to

$$
\tilde{v}_{i}(x)=\tilde{k}_{i} \prod_{j=1}^{n} x_{j}^{\tilde{\sigma}_{i j}}
$$

where the perturbed parameter values $\tilde{k}_{i}$ and $\tilde{\sigma}_{i j}$ are computed as

$$
\tilde{\sigma}_{i j}=\sigma_{i j}+\Delta_{i j}
$$


and

$$
\tilde{k}_{i}=k_{i} \prod_{j=1}^{n} x_{0, j}^{-\Delta_{i j}} .
$$

In addition to the cases where kinetic perturbations can be implemented by changing reaction parameters, a kinetic perturbation $\Delta_{i j}$ can be applied to an arbitrary reaction rate expression $v_{i}(x)$ by the formula

$$
\tilde{v}_{i}(x)=v_{i}(x) x_{j}^{\Delta_{i j}} x_{0, j}^{-\Delta_{i j}} .
$$

An example how a kinetic perturbation changes a linear reaction rate and how that affects the network's dynamics is shown in Figure 1, roughly modelled after example (3) in [21]. Note that while kinetic perturbations which are mapped directly to parameter changes may be implemented in a real biological network by manipulating the corresponding parameter values, the formula (16) is more appropriate for preliminary computational evaluations, when one doesn't want to specify exactly which parameters in a reaction may be modified.

Substituting $\tilde{v}$ for $v$ in the network equations (1), we obtain the perturbed network

$$
\begin{aligned}
\dot{z} & =N_{r} \tilde{v}(x, u) \\
x & =L z+x_{T} \\
y & =K x .
\end{aligned}
$$

In the following section, we study the problem of how to choose $\tilde{v}$, or the perturbation matrix $\Delta$ in (11), such that the pertubed network (17) has perfect adaptation.

\subsection{Computing kinetic perturbations to achieve adapta- tion}

In this section, we develop a method for finding network modifications leading to local adaptation, based on applying kinetic perturbations. Kinetic perturbations are particularly attractive for this problem, as they allow one to keep the network structure unperturbed. Yet, kinetic perturbations can also be used to detect network interactions which can be added or deleted to achieve adaptation, where a network interaction is understood as a link from a species to a reaction whose rate depends on the concentration of this specific species.

By condition (10), kinetic perturbations do not have an effect on the stationary reaction rates $v_{0}$. Thus, it is guaranteed that the steady state $\left(z_{0}, u_{0}\right)$ of the unperturbed network (1) is also a steady state of the perturbed network (17). However, the Jacobian matrix $A=\left.N_{r} \frac{\partial v}{\partial x}\right|_{x_{0}, u_{0}} L$ of the network will in general be affected by the perturbation. In this way, the kinetic perturbation can have an effect on adaptation in the network. Using (11), the perturbed matrix $\tilde{A}$ is computed from a given $\Delta$ as

$$
\begin{aligned}
\tilde{A} & =\left.N_{r} \frac{\partial \tilde{v}}{\partial x}\right|_{x_{0}, u_{0}} L \\
& =A+N_{r} \operatorname{diag} v_{0} \Delta\left(\operatorname{diag} x_{0}\right)^{-1} L .
\end{aligned}
$$

The matrices $B$ and $C$ in (6) are not affected in this setting, although an extended perturbation could be defined which also affects $B$ or $C$. 


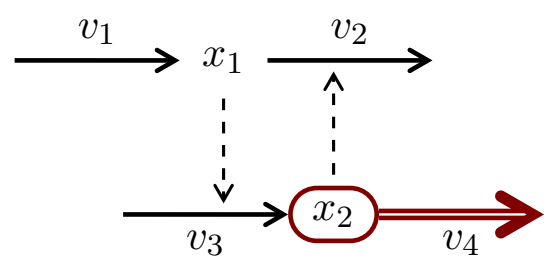

(a) Network structure

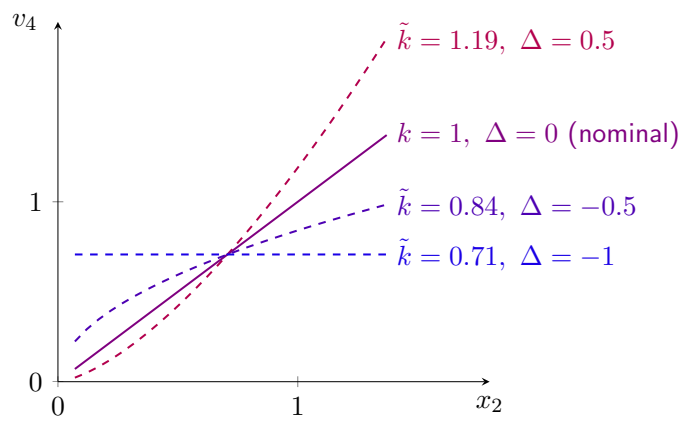

(b) Reaction rate modification

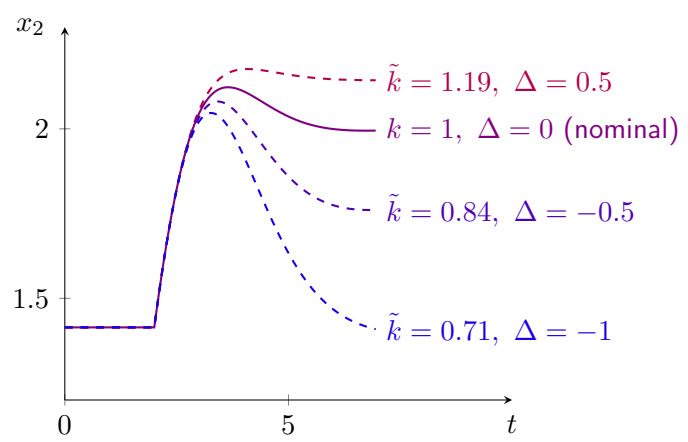

(c) Simulated time courses

Figure 1: Kinetic perturbation of a simple network. (a) Network structure with reaction rates $v_{1}=u, v_{2}=x_{1} x_{2}, v_{3}=0.5 x_{1}, v_{4}=k x_{2}$, with stimulus $u$ and a parameter $k$. Nominal parameters are $u_{0}=1$ and $k=1$. A kinetic perturbation is applied to the specie $x_{2}$ and the reaction $v_{4}$. (b) Dependency of the reaction rate $v_{4}$ on the substrate concentration $x_{2}$. Full line: Reaction rate in the nominal network. Dashed lines: Reaction rate for different kinetic perturbations with $\tilde{v}_{4}=\tilde{k} x_{2}^{1+\Delta}$. (c): Simulation starting at steady state, applying $u=1$ for $t<2$ and $u=2$ for $t \geq 2$. Full line: Time course of the nominal network. Dashed lines: Time courses for the kinetic perturbations as in subfigure (b). 
In order for the perturbed network to have local adaptation, it has to satisfy (9). From (18), this corresponds to the condition

$$
\operatorname{det}\left(\begin{array}{cc}
\tilde{A} & B \\
C & 0
\end{array}\right)=\operatorname{det}\left(\begin{array}{cc}
A+N_{r} \operatorname{diag} v_{0} \Delta\left(\operatorname{diag} x_{0}\right)^{-1} L & B \\
C & 0
\end{array}\right)=0 .
$$

Condition (19) is of the general structure

$$
\operatorname{det}\left(M_{0}+M_{\text {in }} \Delta M_{\text {out }}\right)=0,
$$

with the matrices $M_{0}, M_{i n}$, and $M_{\text {out }}$ defined as follows:

$$
\begin{aligned}
M_{0} & =\left(\begin{array}{cc}
A & B \\
C & 0
\end{array}\right) \\
M_{\text {in }} & =\left(\begin{array}{c}
I_{r} \\
0
\end{array}\right) N_{r} \operatorname{diag} v_{0} \\
M_{\text {out }} & =\left(\operatorname{diag} x_{0}\right)^{-1} L\left(\begin{array}{ll}
I_{r} & 0
\end{array}\right) .
\end{aligned}
$$

Equation (20) is a structured matrix perturbation problem as studied in robust control theory [22, Section 5.1]. It can generally be solved by constructing an appropriate structured singular value problem [23, 22]. While there has been much progress in the past decades to compute $\Delta$ satisfying (20), it may still be challenging for larger networks [22].

Here, we focus on the special case of a scalar kinetic perturbation, where only the effect of a single species $x_{j}$ on a single reaction rate $v_{i}$ is perturbed. Scalar kinetic perturbations are particularly attractive, because they are suitable to achieve adaptation by perturbing a single interaction only, and they allow the ranking of individual interactions with respect to their relevance for adaptation. In the scalar case, $\Delta$ can be written as

$$
\Delta=e_{i} \Delta_{i j} e_{j}^{\mathrm{T}}
$$

where $e_{i} \in \mathbb{R}^{m}$ and $e_{j} \in \mathbb{R}^{n}$ are unit vectors in the direction of the coordinate $i$ and $j$, respectively, and all elements apart from the $(i, j)$ element $\Delta_{i j}$ are zero.

Then, condition (19) becomes

$$
\operatorname{det}\left(\begin{array}{cc}
A+N_{r} \operatorname{diag} v_{0} e_{i} \Delta_{i j} e_{j}^{\mathrm{T}}\left(\operatorname{diag} x_{0}\right)^{-1} L & B \\
C & 0
\end{array}\right)=0 .
$$

Similar to the general case, this is written as

$$
\operatorname{det}\left(M_{0}+M_{\text {in }} e_{i} \Delta_{i j} e_{j}^{\mathrm{T}} M_{\text {out }}\right)=0 .
$$

In the following, we assume that $M_{0}$ is invertible, which by condition (9) means that the network does not yet have local adaptation. The scalar case can be solved without resorting to a reformulation as a structured singular value problem. By applying the Schur complement formula [20, p. 896] twice, (24) is transformed to

$$
1+\Delta_{i j} e_{j}^{\mathrm{T}} M_{\text {out }} M_{0}^{-1} M_{\text {in }} e_{i}=0 .
$$

The solution to (25) is directly computed as

$$
\Delta_{i j}^{*}=\left(-e_{j}^{\mathrm{T}} M_{\text {out }} M_{0}^{-1} M_{\text {in }} e_{i}\right)^{-1} .
$$


This $\Delta_{i j}^{*}$ is the candidate kinetic perturbation in the interaction from species $j$ to reaction $i$ for achieving local adaptation.

It remains to check that applying the obtained kinetic perturbation to the original network (1) does not make the steady state $\left(z_{0}, u_{0}\right)$ unstable, which also includes the condition that the Jacobian $\tilde{A}$ of the perturbed network satisfies $\operatorname{det} \tilde{A} \neq 0$, which was required to derive the adaptation condition (9). We suppose that the steady state $\left(z_{0}, u_{0}\right)$ is exponentially stable in the unperturbed network (1), i.e. the matrix $A$ has all its eigenvalues in the left half complex plane. With the kinetic perturbation $\Delta_{i j}^{*}$ as defined above, the Jacobian of the perturbed network becomes

$$
\tilde{A}=A+N_{r} \operatorname{diag} v_{0} e_{i} \Delta_{i j}^{*} e_{j}^{\mathrm{T}}\left(\operatorname{diag} x_{0}\right)^{-1} L .
$$

For stability analysis, it is useful to define the transfer function $H(s)$ as

$$
H(s)=e_{j}^{\mathrm{T}}\left(\operatorname{diag} x_{0}\right)^{-1} L\left(s I_{r}-A\right)^{-1} N_{r} \operatorname{diag} v_{0} e_{i} .
$$

We can then derive a stability condition for the perturbed network: based on the results in [16], from the argument principle, the perturbed Jacobian $\tilde{A}$ will have all its eigenvalues in the left half complex plane, if

$$
-\Delta^{-}<\Delta_{i j}^{*}<\Delta^{+}
$$

with

$$
\begin{aligned}
& \Delta^{-}=(\sup (\{|H(j \omega)| \mid \omega \in \mathcal{R}, H(j \omega)<0\} \cup\{0\}))^{-1} \\
& \Delta^{+}=(\sup (\{|H(j \omega)| \mid \omega \in \mathcal{R}, H(j \omega)>0\} \cup\{0\}))^{-1},
\end{aligned}
$$

where $\mathcal{R}$ is the realness locus of $H$, that is the (usually finite) set of all frequencies $\omega$ where $H(j \omega)$ is a real number. Note that condition (29) will always fail if the perturbation $\Delta_{i j}^{*}$ induces a zero eigenvalue within an unobservable part of the network. In this case, the perturbed network will not be exponentially stable. Also, a pole-zero cancellation will occur at the origin in the transfer function $G(s)$ of the perturbed network, and the perturbed network will not be adaptive. This problem can usually be counteracted by introducing an additional interaction from the unobservable part of the network to the observable part (see also the examples in Section 3).

In conclusion, under the assumption that the network does not yet adapt, i. e. $M_{0}$ is invertible, we obtain from the analysis of the linear approximation (5) a candidate kinetic perturbation $\Delta_{i j}^{*}$. If $\Delta_{i j}^{*}$ satisfies (29), applying it as kinetic perturbation to the original network (1) will result in a perturbed network (17) having local adaptation at $\left(z_{0}, u_{0}\right)$. Moreover, $(29)$ also guarantees that $\left(z_{0}, u_{0}\right)$ will remain exponentially stable in the perturbed network, and that no pole-zero cancellations occur for $s=0$, which would preclude adaptation.

\subsection{An algorithm to achieve adaptation via kinetic per- turbations}

In this section, we develop an algorithm to construct kinetic perturbations yielding adaptation, based on the theoretical results from the previous sections. For simplicity of presentation, we consider only the scalar case.

The procedure is as follows: 
1. Steady state: Compute a steady state $\left(z_{0}, u_{0}\right)$ and the corresponding matrices $M_{0}, M_{i n}$, and $M_{\text {out }}$ according to (21).

2. Candidate perturbations for adaptation: Iterate over all pairs $(i, j)$ with $i \in\{1, \ldots, m\}$ and $j \in\{1, \ldots, n\}$ and compute the critical value $\Delta_{i j}^{*}$ for the kinetic perturbation according to (26).

3. Pre-check of candidate perturbations: From the list of the resulting $\Delta_{i j}^{*}$, select those that

- have an absolute value smaller than a defined threshold.

- do not violate the stability constraint (29).

4. Detailed analysis of candidate perturbations: For each interaction $(i, j)$ where $\Delta_{i j}^{*}$ satisfies the above conditions, change the reaction rate $v_{i}$ to $\tilde{v}_{i}$, using for example the formula (16) or parameter changes in suitable reaction rate expressions. Evaluate adaptation in the perturbed network (17) for larger stimulus changes, for example by simulation of a step response.

The threshold in step 3 is to assure that very large perturbations, which can be biochemically unrealistic, are not considered further. Recalling that $\Delta_{i j}$ is the normalized change in the slope of reaction rate $v_{i}$ with respect to species concentration $x_{j}$, a threshold on the order of about 1 is usually reasonable. The a posteriori evaluation in step 4 is necessary, because kinetic perturbations yield only local adaptation to infinitesimally small stimulus steps $u_{s}$, and for finite steps the behaviour of the perturbed network has to be evaluated separately.

\section{Examples of local adaptation via kinetic per- turbations}

\subsection{A simple three enzyme network}

Network description As a first example, we consider a simple enzymatic network with three enzymes $X_{A}, X_{B}$, and $X_{C}$ (Figure 2A). Each of the enzymes is assumed to exist in an active and an inactive form. The example is modeled after the analysis in [8], where adapation in enzymatic networks with three enzymes has been analyzed via extensive parameter sampling. The network's output is the activity of the enzyme $X_{C}$, and the stimulus acts on enzyme $X_{A}$. For the network model according to (1), the species concentrations are $x_{1}$ inactive $X_{A}, x_{2}$ - active $X_{A}, x_{3}$ - inactive $X_{B}, x_{4}$ - active $X_{B}, x_{5}$ - inactive $X_{C}, x_{6}$ - active $X_{C}$. The stoichiometric matrix $N_{r}$ and the link matrix $L$ are given by

$$
N_{r}=\left(\begin{array}{cccccc}
1 & -1 & 0 & 0 & 0 & 0 \\
0 & 0 & 1 & -1 & 0 & 0 \\
0 & 0 & 0 & 0 & 1 & -1
\end{array}\right) \quad L=\left(\begin{array}{ccc}
-1 & 0 & 0 \\
1 & 0 & 0 \\
0 & -1 & 0 \\
0 & 1 & 0 \\
0 & 0 & -1 \\
0 & 0 & 1
\end{array}\right)
$$




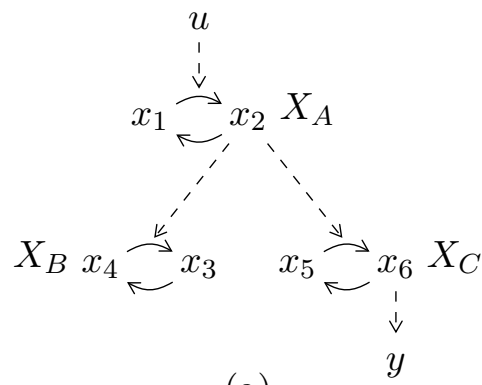

(a)

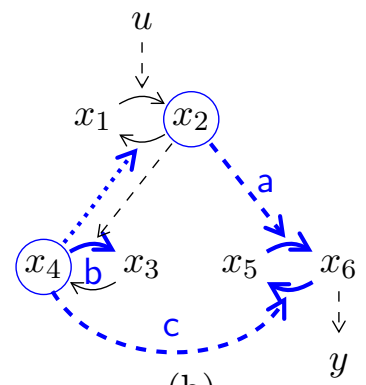

(b)

Figure 2: Interaction structures and candidate interactions for adaptation in the simple three enzyme network. (a) Base network structure. (b) Base structure with candidate interactions for adaptation (species encircled and reactions as thick blue lines) as obtained from the analysis with kinetic perturbations. For each group labelled a,b,c in Table 1, one candidate interaction is shown. Note that networks perturbed via a or $\mathrm{b}$ retain the original network structure, while the perturbation via $c$ introduces a new interaction into the network. Dotted line: Additional feedback interaction required to maintain stability with perturbation b. (Color online.)

and the vector of total concentrations is chosen as

$$
x_{T}=(1,0,1,0,1,0)^{\mathrm{T}} .
$$

The reaction rate expressions are given as follows:

$$
\begin{aligned}
v_{1} & =\frac{u x_{1}}{1+x_{1}} & v_{2} & =\frac{0.5 x_{2}}{1+x_{2}} \\
v_{3} & =\frac{0.5 x_{3}}{1+x_{3}} & v_{4} & =\frac{x_{2} x_{4}}{1+x_{4}} \\
v_{5} & =\frac{x_{2} x_{5}}{1+x_{5}} & v_{6} & =\frac{0.5 x_{6}}{1+x_{6}} .
\end{aligned}
$$

In the next paragraphs, we describe the application of the algorithm from Section 2.4 to achieve adaptation in this network.

Step 1: A steady state of the three enzyme network is computed with nominal stimulus $u_{0}=1$. This steady state is characterized by $x_{2}=0.73, x_{4}=0.36$, and $x_{6}=0.64$. Also the matrices $M_{0}, M_{i n}$, and $M_{\text {out }}$ are computed, but for brevity not shown here.

Step 2: The candidate interactions, where a kinetic perturbation may lead to perfect adapation, are computed by (26) and are given in Table 1 . Note that due to symmetries in the network structure, we can identify groups of perturbations having the same effect on the linearized dynamics. For each group of perturbations, one representative interaction to be perturbed is shown in Figure 2B. 


\begin{tabular}{|l|ll|r|}
\hline & Enzyme & Reaction & $\Delta_{i j}^{*}$ \\
\hline \hline \multicolumn{3}{|c|}{ a) Interactions from $X_{A}$ to $X_{C}$} \\
\hline & $X_{A}$ active & $X_{C}$ activation & -1.00 \\
& $X_{A}$ active & $X_{C}$ inactivation & 1.00 \\
& $X_{A}$ inactive & $X_{C}$ activation & 0.37 \\
& $X_{A}$ inactive & $X_{C}$ inactivation & -0.37 \\
\hline \hline \multicolumn{3}{|c|}{ b) Interactions from $X_{B}$ to $X_{B}$} \\
\hline & $X_{B}$ active & $X_{B}$ activation & $1.08^{*}$ \\
& $X_{B}$ active & $X_{B}$ inactivation & $-1.08^{*}$ \\
& $X_{B}$ inactive & $X_{B}$ activation & $-1.90^{*}$ \\
& $X_{B}$ inactive & $X_{B}$ inactivation & $1.90^{*}$ \\
\hline \hline \multicolumn{5}{|c|}{ Interactions from $X_{B}$ to $X_{C}$} \\
\hline & $X_{B}$ active & $X_{C}$ activation & 1.90 \\
& $X_{B}$ active & $X_{C}$ inactivation & -1.90 \\
& $X_{B}$ inactive & $X_{C}$ activation & -1.08 \\
& $X_{B}$ inactive & $X_{C}$ inactivation & 1.08 \\
\hline
\end{tabular}

Table 1: Candidate perturbations for adaptation in the three enzymes model. Entries in each group have the same effect on the matrix $M_{0}$. Perturbations marked with * yield a zero eigenvalue in the network's Jacobian.

Step 3: Due to the simplicity of this example, it is not necessary to apply a threshold on the perturbation strength. We just keep all candidates. However, the perturbations from group b (marked with * in Table 1 ) fail the stability test (29). In fact, the perturbed Jacobian has an eigenvalue at zero. A pole/zero cancellation will occur in the transfer function $G(s)$, precluding adaptation.

This problem can be avoided by adding a feedback from $X_{B}$ to $X_{A}$, which is achieved by the kinetic perturbation

$$
\tilde{v}_{1}=\frac{x_{1}}{1+x_{1}} x_{0,4}^{-1} x_{4},
$$

With the integral behaviour in $X_{B}$ from the suggested kinetic perturbations in Table 1b), this becomes an integral feedback, a well known motif for biochemical adaptation [10].

Step 4: Let us first consider the group of perturbations labelled a in Table 1. These perturbations remove the effect of $X_{A}$ activity on the change of $X_{C}$ activity. This corresponds to a disconnection of $X_{C}$ activity, and thus the output, from $X_{A}$, where the input acts. In consequence, the steady state output of the perturbed network does not change under stimulus variations, but there is also a lack of the transient response.

While kinetic perturbations from the group $\mathrm{b}$ in Table 1 improve adaptation compared to the nominal network, no perfect adaptation is obtained. This is due to the restriction that the proposed kinetic perturbations yield local adaptation only. In such a case, using a different value for the perturbation $\Delta_{i j}$ may decrease the adaptation error as shown in Figure 3.

Finally, let us consider the group of perturbations labelled c in Table 1. The resulting perturbed network is an incoherent feedforward loop: $X_{A}$ activates 


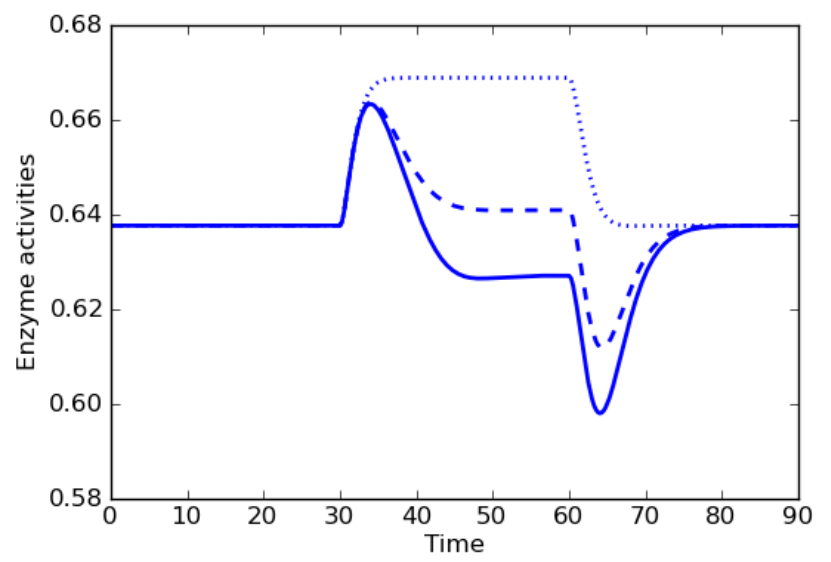

Figure 3: Output time courses for adaptation in the feedback network structure. Original and perturbed networks are simulated from steady state with an input of $u=1$ during the first interval of 30 time units, $u=1.5$ during the second interval of 30 time units, and $u=1$ during the third interval of 30 time units. Dotted line: original network; full line: network with kinetic perturbation $\Delta_{44}^{*}=$ -1.08; dashed line: network with kinetic perturbation $\Delta_{44}=-1.0$.

$X_{C}$ directly, but inhibits it indirectly via $X_{B}$. The incoherent feedforward loop is another classical example of adaptive network motifs [8]. Time courses for two different perturbations in this group are shown in Figure 4, also in comparison with the output time course of the original network. Both perturbations show near perfect adaptation also for large changes in the input.

In summary, the analysis of a basic three enzyme network with kinetic perturbations has revealed all the possibilities for adaptation which have previously been found only by an extensive computational parameter scan study [8]. In the case of adaptation via integral feedback, the approach revealed that feedback from $X_{B}$ to $X_{A}$ is necessary to also satisfy the stability constraint.

\subsection{Adaptation in the MAPK cascade}

The second case study is to search for modifications yielding adaptation in a mitogen activated protein kinase (MAPK) cascade. For brevity, we will not discuss the biological details and implications of adaptation in the MAPK cascade, but merely consider this system as a case study where adaptation through kinetic perturbations is evaluated in a medium-size signaling network model.

The analysis is based on the model of the MAPK cascade as presented in [24]. This model is built from a detailed biochemical description of reactions in the MAPK pathway. All enzymatic reactions are modeled with the Michaelis-Menten mechanism, using mass action kinetics and including intermediate enzyme-substrate complexes explicitly in the model. We make two extensions to the original model from [24] which are appropriate in the context of achieving adaptation. First, to fit the framework outlined in Section 2.1, activation and deactivation of the enzyme E1 at the top of the cascade is added to the model. This extension provides a control parameter $u$ which is in the 


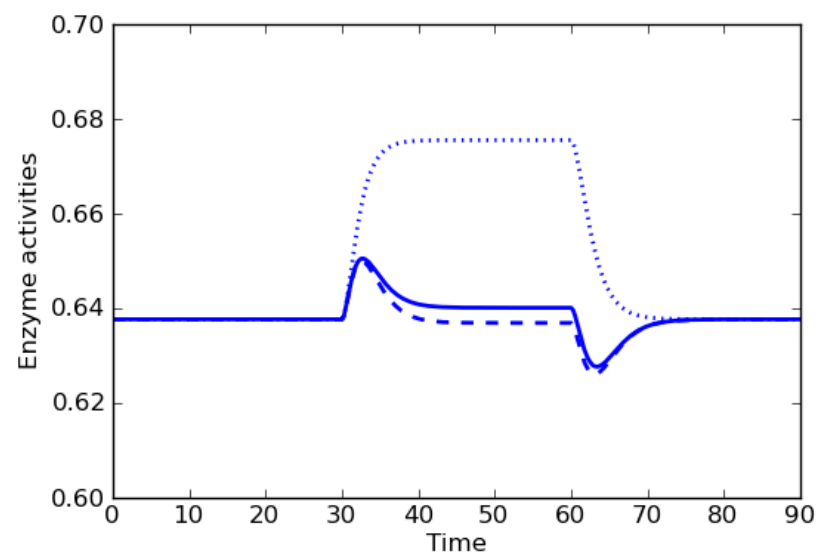

Figure 4: Output time courses for adaptation in the feedforward network structure. Original and perturbed networks are simulated from steady state with an input of $u=1$ during the first interval of 30 time units, $u=1.5$ during the second interval of 30 time units, and $u=1$ during the third interval of 30 time units. Dotted line: original network; dashed line: adaptation via interaction from active $X_{B}$ to activation of $X_{C}, \Delta_{54}^{*}=1.90$; full line: adaptation via interaction from inactive $X_{B}$ to activation of $X_{C}, \Delta_{53}^{*}=-1.08$.

following considered as the stimulus. Second, we add a model part describing expression of a protein regulated by MAPK, shortly termed RP. This extension is done because the original network cannot be made adaptive by scalar kinetic perturbations, as an application of the algorithm proposed in Section 2.4 to this network showed. Intuitively, this is due to the lack of potential buffering or proportioner nodes, which are a known prerequisite for adaptation [8]. The structure of the model with these extensions is shown in Figure 5. The full model definition including parameters and initial conditions is provided in an SBML file as supplementary online information. In Figure 5 and Table 2, MAPK, MAPKK, and MAPKKK are abbreviated as K, KK, and KKK, respectively.

The goal of this case study is to find kinetic perturbations that yield adaptation of the nuclear MAPKpp activity to variations in the stimulus $u$. We first compute the critical $\Delta_{i j}^{*}$ for all species-reaction interactions in the model. A cutoff threshold $\Delta_{i j}^{*} \leq 1$ is chosen to restrict the number of resulting candidate interactions to a reasonable amount. The results are given in Table 2. The first five candidate perturbations concern interactions within the direct path from the stimulus to the output. Already from this observation, we can suspect that applying these perturbations could lead to a total interruption of signal flow and loss of the transient response to changes in the stimulus, and this has been confirmed by simulating the perturbed systems. For the remaining four candidate perturbations, a pole-zero cancellation problem for the perturbed systems is detected, thus precluding adaptation. This is also intuitively clear from the observation that these perturbations affect only the non-observable part of the model.

To avoid the problem with pole-zero cancellations, we add a feedback from the non-observable part to the observable part of the system, similarly as has 


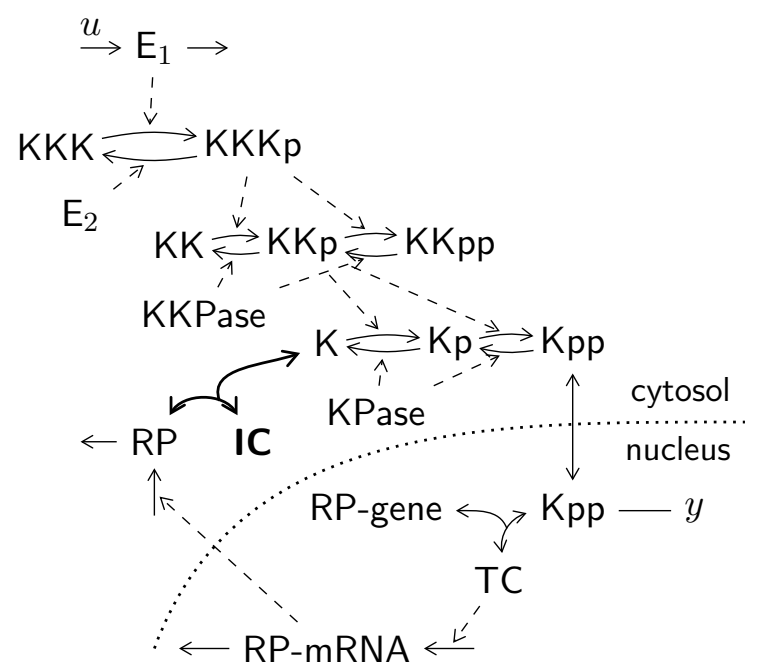

Figure 5: Structure of the MAPK cascade model. The specie IC and associated reactions (shown in blue) are only included in the model variant with feedback. The model variant without feedback has 27 species and 40 reactions. To keep the illustration concise, enzyme-substrate complexes are not shown, although they are explicitly included in the model's equations.

\begin{tabular}{|ll|c|c|}
\hline Species & Reaction & $\left.\Delta_{i j}^{*} \mathrm{a}\right)$ & $\left.\Delta_{i j}^{*} \mathrm{~b}\right)$ \\
\hline \hline E1 & binding of E1 to KKK & -1 & -1 \\
E1 & binding of E2 to KKKp & 1 & 1 \\
E1/KKK complex & complex dissociation & -1 & -1 \\
Kpp & nuclear import of Kpp & -1 & -1 \\
Kpp & nuclear export of Kpp & 1 & 1 \\
RP-mRNA & degradation of RP-mRNA & $-1^{*}$ & -1 \\
RP & degradation of RP-mRNA & $-1^{*}$ & -1 \\
RP & translation of RP & $1^{*}$ & 1 \\
RP & degradation of RP & $-1^{*}$ & -1 \\
\hline
\end{tabular}

Table 2: Candidate perturbations in MAPK. Entries marked with * don't satisfy the stability condition (29), and lead to a pole-zero cancellation and no adaptation as discussed in Section 2.1. a) Critical $\Delta_{i j}$ values in model without feedback from RP to MAPK. b) Critical $\Delta_{i j}$ values in model with feedback from RP to MAPK via complex IC (see Figure 5). 


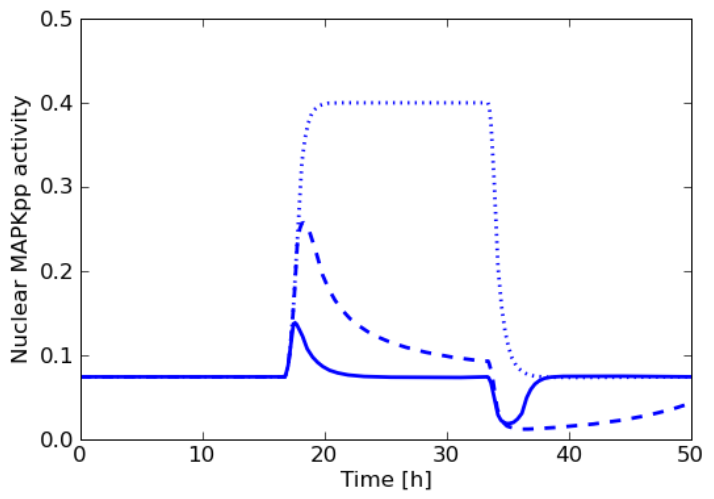

Figure 6: Comparison of stimulus response dynamics in the nominal and perturbed MAPK models with feedback from RP to MAPK. Models were simulated from the unstimulated steady state with $u=2 \cdot 10^{-6}$ for 1000 minutes, $u=3 \cdot 10^{-6}$ for another 1000 minutes, and $u=2 \cdot 10^{-6}$ for a third time interval of 1000 minutes, Dotted line: nominal model. Dashed line: model with kinetic perturbation $\Delta^{*}=-1$ in the interaction from RP to the degradation of RP. Full line: model with kinetic perturbation $\Delta^{*}=-1$ in the interaction from RP to the degradation of RP mRNA.

been done in the three enzymes example (Section 3.1). In this study, such a feedback is achieved by postulating that the regulated protein RP binds to unphosphorylated MAPK and inhibits its phosphorylation. This inhibitory complex of RP and unphosphorylated MAPK is denoted as IC in Figure 5.

We again search candidate perturbations for adaptation in the model with feedback. The results are given in Table 2, again using the threshold $\Delta_{i j}^{*} \leq 1$. As previously, the first five perturbations lead to an interruption of signal flow and a lack of the transient response. With the other four perturbations, it is in fact possible to achieve perfect adaptation. Time courses showing the response to input steps are shown in Figure 6.

In summary, the proposed approach can also be applied to determine perturbations for adaptation in more complex signaling pathway models like the MAPK cascade.

\section{Discussion and conclusions}

We presented a method to systematically construct an adaptive model from a non-adaptive one. This is achieved by perturbations of the rate expressions such that a transmission zero in the origin is obtained, which is a necessary and sufficient condition for local adaptation. Basically, we proceed as follows: candidate kinetic perturbations are computed for the network using the conditions on local adaptation. The detailed analysis of these candidates then allows a judgement how reaction rates can be changed to obtain adaptation. The method was illustrated with two examples to systematically identify modifications of the network that lead to adaptation. The examples have shown that the method can be used to detect several possibilities for adaptation, and that 
it is applicable to networks of realistic complexity.

The candidate kinetic perturbations are computed from the linear approximation of the network around the considered steady state. Therefore, applying the perturbations to the network only guarantees local adaptation. Yet, as the examples illustrate, they improve adaptive behaviour even to large stimulus changes in most cases, and can even achieve perfect or partial adaptation in many cases.

In comparison to other approaches, our method does not rely on extensive sampling of the possibly multi-dimensional parameter space (e.g. [8]). As an alternative to sampling, also set-based methods can be used for the analysis of adaptation core-motifs [25]. Especially for large networks, sampling and setbased approaches can be computationally demanding and they may therefore only be feasible to analyze core-motifs. However, a search for core-motifs might not be easy and is not always intuitive.

Another advantage is that the stoichiometric matrix is left untouched by the proposed method, but only reaction kinetics and parameters are perturbed. This might be desired because very often the stoichiometry of a network is well known, but the kinetic rate expressions or parameters are not. In relation to the distinction between mass and signal flow made in [26], kinetic perturbations leave the network's mass flow unaffected, but may change the signal flow.

With kinetic perturbations, the network does not necessarily adapt to arbitrarily large changes of the stimulus. Depending on the type of the reaction that needs to be perturbed for adaptation and on the expression for the kinetic perturbation, the achieved adaptation may be local or non-robust. Yet, for certain classes of reaction rates such as generalized mass-action, kinetic perturbations will typically transform a first-order reaction into a zero-order reaction, which is often a robust adaptation mechanism even for large stimulus changes. In any case, kinetic perturbations can be used to systematically detect rates where perturbations are most promising to achieve adaptation.

The method can be particularly useful in iterative model building processes where one model part was previously derived by quantitative fitting to experimental data. The approach intrinsically guarantees that the kinetically perturbed system maintains the same steady state and with the same mass flow as the original system for the nominal stimulus. Therefore, the method can be used to extend the quantitative model core by additional mechanisms that account for the observed adaptation.

An alternative application of the proposed method is in synthetic biology. As discussed in [27], the design of synthetic networks with adaptation is "the next frontier" in metabolic engineering. In large synthetic networks which are assembled from small modular parts, it will become important to achieve a specific overall behaviour, such as adaptation, without perturbing other desired network functions, such as the nominal steady state [28]. In this context, kinetic perturbations may offer a useful tool for the fine-tuning of large synthetic biomolecular networks.

\section{Acknowledgement}

We thank Philipp Rumschinski, Daniella Schittler, and Patrick Weber for comments on the manuscript. The authors S.W. and F.A. would like to thank 
the German Research Foundation (DFG) for financial support of the project within the Cluster of Excellence in Simulation Technology (EXC 310/1) at the University of Stuttgart.

\section{References}

[1] Levchenko, A., and Iglesias, P.A.: 'Models of eukaryotic gradient sensing: application to chemotaxis of amoebae and neutrophils', Biophys. J., 2002, 82 (1 Pt 1), pp. 50-63

[2] Wadhams, G.H., and Armitage, J.P.: 'Making sense of it all: bacterial chemotaxis', Nat. Rev. Mol. Cell Biol., 2004, 5 (12), pp. 1024-1037

[3] Streif, S., Oesterhelt, D., and Marwan, W.: 'A predictive computational model of the kinetic mechanism of stimulus-induced transducer methylation and feedback regulation through $\mathrm{CheY}$ in archaeal phototaxis and chemotaxis', BMC Syst. Biol., 2010, 4, 27

[4] Muzzey, D., Gómez-Uribe, C.A., Mettetal, J.T., and van Oudenaarden, A.: 'A systems-level analysis of perfect adaptation in yeast osmoregulation', Cell, 2009, 138 (1), pp. 160-171

[5] Drengstig, T., Ueda, H.R., and Ruoff, P.: 'Predicting perfect adaptation motifs in reaction kinetic networks', J. Phys. Chem. B, 2008, 112 (51), pp. $16752-16758$

[6] Barkai, N., and Leibler, S.: 'Robustness in simple biochemical networks', Nature, 1997, 387 (6636), pp. 913-917

[7] Marwan, W., Bibikov, S.I., Montrone, M., and Oesterhelt, D.: 'Mechanism of photosensory adaptation in Halobacterium salinarium', J. Mol. Biol., 1995, 246 (4), pp. 493-499

[8] Ma, W., Trusina, A., El-Samad, H., Lim, W.A., and Tang, C.: 'Defining network topologies that can achieve biochemical adaptation', Cell, 2009, 138 (4), pp. $760-773$

[9] Sontag, E.: 'Adaptation and regulation with signal detection implies internal model', Syst. \& Contr. Lett., 2003, 50 (2), pp. 119-126

[10] Yi, T.M., Huang, Y., Simon, M.I., and Doyle, J.: 'Robust perfect adaptation in bacterial chemotaxis through integral feedback control', Proc. Natl. Acad. Sci. U. S. A., 2000, 97 (9), pp. 4649-4653

[11] Yi, T.M., Andrews, B.W., and Iglesias, P.A.: 'Control analysis of bacterial chemotaxis signaling', Meth. Enyzmol., 2007, 422, pp. 123-140

[12] Ni, X.Y., Drengstig, T., and Ruoff, P.: 'The control of the controller: molecular mechanisms for robust perfect adaptation and temperature compensation', Biophys. J., 2009, 97 (5), pp. 1244-1253

[13] Rao, C.V., Kirby, J.R., and Arkin, A.P.: 'Design and diversity in bacterial chemotaxis: a comparative study in Escherichia coli and Bacillus subtilis', PLoS Biol., 2004, 2 (2), e49 
[14] Kollmann, M., Løvdok, L., Bartholomé, K., Timmer, J., and Sourjik, V.: 'Design principles of a bacterial signalling network', Nature, 2005, 438 (7067), pp. 504-507

[15] Ang, J., Bagh, S., Ingalls, B.P., and McMillen, D.R.: 'Considerations for using integral feedback control to construct a perfectly adapting synthetic gene network', J. Theor. Biol., 2010, 266 (4), pp. 723-738

[16] Waldherr, S., Allgöwer, F., and Jacobsen, E.W.: 'Kinetic perturbations as robustness analysis tool for biochemical reaction networks', in Proc. of the 48th Conf. Dec. Contr. (CDC) (Shanghai, China, 2009), pp. 4572-4577

[17] Heinrich, R., and Schuster, S.: The Regulation of Cellular Systems (Chapman \& Hall, New York, 1996)

[18] Ingalls, B.: 'A frequency domain approach to sensitivity analysis of biochemical networks', J. Phys. Chem. B, 2004, 108 (3), pp. 1143-52

[19] Kuznetsov, Y.A.: Elements of Applied Bifurcation Theory (SpringerVerlag, New York, 1995)

[20] Ogata, K.: Modern Control Engineering (Prentice-Hall, Englewood Cliffs, USA, 1990), 2nd edn.

[21] Steuer, R., Waldherr, S., Sourjik, V., and Kollmann, M.: 'Robust signal processing in living cells', PLoS Comp. Biol., 2011, 7 (11), e1002218

[22] Hinrichsen, D., and Pritchard, A.J.: Mathematical Systems Theory I (Springer-Verlag, Berlin, 2005)

[23] Doyle, J.: 'Analysis of feedback systems with structured uncertainties', IEE Proc. Contr. Theo. Appl., 1982, 129 (6), pp. 242-250

[24] Huang, C.Y., and Ferrell, J.E.: 'Ultrasensitivity in the mitogen-activated protein kinase cascade', Proc. Natl. Acad. Sci. U. S. A., 1996, 93 (19), pp. $10078-83$

[25] Rumschinski, P., Streif, S., and Findeisen, R.: 'Combining qualitative information and semi-quantitative data for guaranteed invalidation of biochemical network models', Int. J. Robust Nonlin. Control, 2012, advanced online publication

[26] Klamt, S., Saez-Rodriguez, J., Lindquist, J., Simeoni, L., and Gilles, E.D.: 'A methodology for the structural and functional analysis of signaling and regulatory networks', BMC Bioinformatics, 2006, 7, 56

[27] Holtz, W.J., and Keasling, J.D.: 'Engineering static and dynamic control of synthetic pathways', Cell, 2010, 140 (1), pp. 19-23

[28] Lu, T.K., Khalil, A.S., and Collins, J.J.: 'Next-generation synthetic gene networks', Nat. Biotechnol., 2009, 27 (12), pp. 1139-1150 